\title{
EDITORIAL
}

\section{The Changing Face of Vaccination}

The term "vaccination" is used throughout this article as a synonym for the more correct but unattractive term "immunisation". Louis Pasteur initiated this usage when he named his immunogenic attenuated anthrax culture a "vaccine" in honour of Edward Jenner's pioneering work on smallpox.

In 1978 the United Kingdom was a signatory to the Declaration of Alma Ata, in which the World Health Organisation set out goals for the attainment of "Health for All by the Year 2000". In 1985 the European office of WHO defined agreed targets for the countries of the Region, one of which states, "By the year 2000, there should be no indigenous measles, poliomyelitis, neonatal tetanus, congenital rubella, diphtheria, congenital syphilis or indigenous malaria in the region".

The publication of an intention to eradicate communicable disease on such a scale reflects a confidence in modern vaccination technology and health promotion techniques which would not have existed a decade ago. The United Kingdom has taken up the challenge with the campaign to eliminate measles, mumps and rubella with the introduction of a combined Measles/Mumps/Rubella (MMR) Vaccine on 1 October 1988.

The DHSS have forwarded full details of the new vaccine to all doctors, together with an extensively revised copy of the Joint Committee on Vaccination and Immunisation Handbook, "Immunisation against Infectious Disease". These and other changes in Service vaccination policy have necessitated a complete revision of the Tri-Service Memorandum on Immunological Procedures, JSP 311, which is now under way. At the end of this process the long established and familiar schedule of DTP, Poliomyelitis, TABT, Smallpox and BCG vaccination dating from the post war years will have altered radically with the removal of familiar vaccines and addition of new ones.

The early history of vaccination procedures is full of interest for the Army Medical Officer in view of the early contributions of Wright, Bruce, Leishman and many others. However, a very brief review of developments since the Second World War is more relevant to consideration of the changes now taking place.

Smallpox was the first of the long established vaccination procedures to be discontinued. Early attempts by WHO to control smallpox by mass vaccination had failed but a new initiative based on contact tracing and selective vaccination was launched in 1967. This reached a triumphant conclusion in 1979 when the world was declared free of smallpox. This declaration was ratified at the 33rd World Health Assembly in May 1980. Smallpox vaccination of Service dependents was discontinued, but remained in the schedule for Service personnel until 1982. It is of interest to note that even for a disease as feared as smallpox the infant vaccination rate for many years was of the order of 40 per cent.

Diphtheria vaccination was introduced on a national scale in 1941 and resulted in a rapid and dramatic fall in the number of notifications and deaths from the disease. In 1940 there were 2,480 deaths. In 1953 this had fallen to 23 , and only one death was recorded between 1979 and 1986. Although the organism has been virtually eliminated in the United Kingdom, it is essential to maintain a high vaccine acceptance rate in children to prevent spread of disease from a chance importation.

Tetanus vaccination was introduced routinely into the primary vaccination schedule for infants in 1961. Such vaccinations are given for the benefit of the individual as there is no elimination of Clostridium tetani or herd immunity effect. The aim is now to achieve universal active immunity allowing the use of a reinforcing dose of toxoid in the management of a tetanus prone wound. I I the past, tetanus vaccination of Service personnel under 35 years of age was linked with the use of TAB vaccine and carried out at 3 year intervals. An interval of 5 yearo or longer is desirable to reduce the risk of hyper sensitivity reactions, and Service policy is likely t\$ change with the introduction of monovalent typhoid vaccine.

Whooping cough vaccine, the third element of the DTP “Triple Vaccine", was introduced in 1957 but ver soon became the subject of controversy. At the beginning there was no sudden drop in the mortality trend comparable with that for diphtheria, and later studies suggested that the protection rate was considerably less than had been claimed. At the same time the number of adverse reactions was in excess of earlier predictions. The situation improved with the introduction of a more effective vaccine in 1969. However, a number of reports appeared which suggested that the incidence of severe reactions, including permanent brain damage, was unacceptable and that the use of this vaccine should be discontinued.

Medical calamity generates political and media interest far greater than medical success, and these reports were taken up enthusiastically by the press. The acceptance rate for whooping cough vaccination fell from 80 per cent in 1974 to only 31 per cent in 1978. In response to public concern and lack of reliable quantitative data on side effects the National Child Encephalopathy Study was set up in 1976 to report on the safety of the vaccine with particular reference to brain damage. The nature of the data available did not allow a precise assessment of risk, but the study estimated the attributable risk of persistent brain 
damage in previously normal children, following a full primary course of vaccine, to be of the order of 1 in 310,000. The Joint Committee on Vaccination and Immunisation then reviewed all the available evidence and concluded that the current vaccine is protective and that its use should be continued. The acceptance rate rose to over 70 per cent by 1987 . Work is now in progress on new acellular vaccines which may have fewer side effects and be more acceptable.

At the present time BCG vaccination is still recommended for all children aged between 10 and 13 years. It has been estimated that 10,000 such vaccinations will prevent only one case in the following 10 years. The case for routine vaccination will be reviewed by the DHSS in 1990, and it is probable that the decision will be made to introduce selective vaccination in England and Wales from that date.

The WHO aim to eliminate paralytic poliomyelitis from Europe has virtually been achieved in the United Kingdom following the introduction of the Salk inactivated poliomyelitis vaccines (IPV) in 1956 and of the Sabin oral poliomyelitis vaccine (OPV) in 1962. The average number of such cases has fallen to only 2 per year since 1980. Consideration has been given to primary vaccination with IPV followed by OPV in order to eliminate the very small risk of vaccine associated cases resulting from the use of the live virus, but at present IPV is used only in exceptional circumstances.

From 1 October 1988, combined MMR vaccine will replace the measles vaccine in use since 1968 , reinforce rubella vaccination in use since 1970 , and add a mumps vaccine component. In the USA mumps vaccine has 흘 been in use for many years and has greatly reduced the number of cases and complications. The new vaccine will be given to children of both sexes aged 1 to 2 years, and to children aged 4 to 5 years at the time of the preschool diphtheria, tetanus and poliomyelitis booster. It should be emphasized that the aim of this historic initiative is the elimination of the three diseases. This will require the very high acceptance rate of 90 per cent which is appreciably more than the current acceptance rate of 84 per cent for diphtheria vaccine.

\section{NOTICE TO SUBSCRIBERS}

Space orders should be sent to Combined Service Publications Ltd., P.O. Box 4, Farnborough, Hampshire GU14 7LR. Artwork and/or Copy should be forwarded to the same address to arrive by the first day of the month preceding issue, publication dates being February, June and October of each year.

Advertising Rates and technical Details are as listed in British Rate and Data. 